\title{
The research on best strategy of adding hot water
}

\author{
Lei Xing ${ }^{1, a}$ \\ ${ }^{1}$ North China Electric Power University, Baoding 071003, China; \\ a15733230658@163.com
}

Keywords: Temperature model, Heat transfer coefficient, Heat balance

\begin{abstract}
In this paper we select a set of parameters to analyst the warming and cooling progress of the water in the bathtub and view the moment of adding hot water as the main standard for our evaluation. Based on Law of conservation of energy and Newton's law of cooling, the temperature model was established. The loss heat of water which is due to the heat exchange between water and air is the key. We derive a differential equation for it. Then, we find that adding hot water at 115 s per cycle will lead to the least water wasting which is about $249 \mathrm{~kg}$. The result states that adding water too early or too late is not a sensible choice. The outcome of sensitive analyst about the flow rate displays that the effect of it on the model is small.
\end{abstract}

\section{Introduction}

The background of the problem is a person fills a bathtub with hot water from a single faucet and settles into the bathtub to cleanse and relax. Unfortunately, the bathtub is a simple water containment vessel, which means that the bathtub doesn't have an automatic control system.

The person having a hot bath in the bathtub has to add hot water to reheat the bathing water by himself. The source of the hot water is a constant trickle of hot water from the faucet. The bathtub is designed in such a way that when the tub reaches its capacity, excess water escapes through an overflow drain.

\section{Model Overview}

In the basic model, the change of the temperature is considered to meet the demand of Newton's law of cooling, that is to say, the association between the temperature of the water in the bathtub and the time meets the index law.

For the first, we focus on the conversion and conservation of energy. The loss of water energy in the bathtub due to the heat exchange between the surface of water and air is the key. We derive a differential equation for it.

Next, we discuss which time is the right moment to add a constant trickle of hot water from the faucet to reheat the bathing water. Since different people have various suitable temperature they can accept and there also exists a minimum temperature which is supposed to add hot water, it is necessary to take them in to account. After deriving an expression for both cases, we can find that to waste water as little as possible, users are ought to add hot water when the temperature decline to the extent which they can endure.

\section{The Model}

Step 1. The establish of cooling model

Notice that the change of water temperature follows Newton's law of cooling[1], so the relation between the water temperature and time is displayed as follows:

$$
T=a e^{b t}
$$

Since we don't need to worry about the shape and volume of the bathtub in the basic model, we simplify the bathtub into an idealized model. We assume that the shape of the bathtub is cuboid, and 
the length of upper surface of bathtub is $0.75 \mathrm{~m}$, while the width is $0.8 \mathrm{~m}$, so the area of contact surface between the water and air is $0.6 \mathrm{~m} 2$.

Following the fact that, the best temperature when people take a hot bath is $42{ }^{\circ} \mathrm{C}$, and we assume that as the time goes by 150 seconds, the temperature of the water in the bathtub will decline to $33^{\circ} \mathrm{C}$, which is the minimum temperature users need to add hot water.

Based on the information shown above, it is not difficult to conclude the model which reflects the change of water temperature:

Step 2. The establish of heating model

$$
T=42 e^{-0.0016 t}
$$

According to the assumptions mentioned above, it is easily to find that the model we build follows the law of conservation of energy[2].

$$
Q_{\text {in }}=Q_{\text {out }}
$$

In the process of heat exchange, the system has absorbed about $\triangle \mathrm{Q}$ heat, when the temperature rises up $\triangle T$, so the thermal capacity in the process is shown as follows:

$$
\Delta Q=c m \Delta T
$$

Adding hot water when the water temperature comes down to Tc, which is a random value of number between the initial temperature and the minimum temperature. The moment is tc, and the temperature of hot water is $80^{\circ} \mathrm{C}$. According to hydromechanics, we let the flow rate of incoming hot water is $0.8 \mathrm{~kg} / \mathrm{s}$. By law of conservation of energy, the quantity of heat absorbed is equal to the heat lost.

The quantity of heat absorbed can be expressed

$$
\Delta Q_{1}=c m_{1}\left(T-T_{h}\right)
$$

The quantity of heat lost can be expressed in a similar way

$$
\Delta Q_{2}=c\left(m_{0}-m_{2}\right)\left(T-T_{c}\right)
$$

As we all know, the quality of the hot water added is the same with the overflowing water. There is no deny that

$$
m_{1}=m_{2}=q \Delta t
$$

So we can obtain the connection between Q1and Q2

$$
Q_{1}=-Q_{2}
$$

Consider that the process can be divided into many tiny period of time. We take differentia of the case shown in Eq., the result is

$$
T^{\prime}+\frac{q}{m_{o}} T=\frac{q}{m_{o}} T_{h}
$$

The root of equation is shown below

$$
T=\left(C-T_{h}\right) e^{-\frac{q\left(t-t_{c}\right)}{m_{o}}}+T_{h}
$$

In order to get the constant, we let $t=t_{c}$ in the equation, so that $C=T_{c}$. Finally we obtain the heating model:

$$
T=\left(T_{c}-T_{h}\right) e^{-\frac{q\left(t-t_{c}\right)}{m_{o}}}+T_{h}
$$

Step 3. The minimize of the waste water

Taken the two models mentioned above, we can conclude the expression of the temperature with time and space.

$$
T=\left\{\begin{array}{l}
42 e^{-0.0016 t} \\
\left(T_{c}-T_{h}\right) e^{-\frac{q\left(t-t_{c}\right)}{m_{o}}}+T_{h}
\end{array}\right.
$$




\section{Analysis of the Result}

Firstly, we add hot water when the water temperature comes down to Tcmin, which is the minimum temperature.

$$
T=\left\{\begin{array}{l}
42 e^{-0.0016 t} \\
\left(T_{c}-T_{h}\right) e^{-\frac{q\left(t-t_{c}\right)}{m_{o}}}+T_{h}
\end{array}\right.
$$

where $\mathrm{Tc}$ is $33^{\circ} \mathrm{C}$, tc is $150 \mathrm{~s}$, and we let $\mathrm{m} 0$ represent the quality of water in the bathtub. The result is that the total cooling and heating time per cycle is 192 seconds on the conditions.

$$
T=\left\{\begin{array}{l}
42 e^{-0.0016 t}(0<t \leq 150) \\
\left(T_{c}-T_{h}\right) e^{-\frac{q\left(t-t_{c}\right)}{m_{o}}}+T_{h}(150<t \leq 192)
\end{array}\right.
$$

If the total time users need in a hot bath is 30 minutes, then the total hot water he uses during one cycle is $m_{1}=q\left(t-t_{c}\right)=33.6 \mathrm{Kg}$. After he over the bath, the total hot water is $m_{1}=N q\left(t-t_{c}\right)=9 \bullet 33.6=302.4 \mathrm{Kg}$, where $\mathrm{N}$ is the number of the cycle.

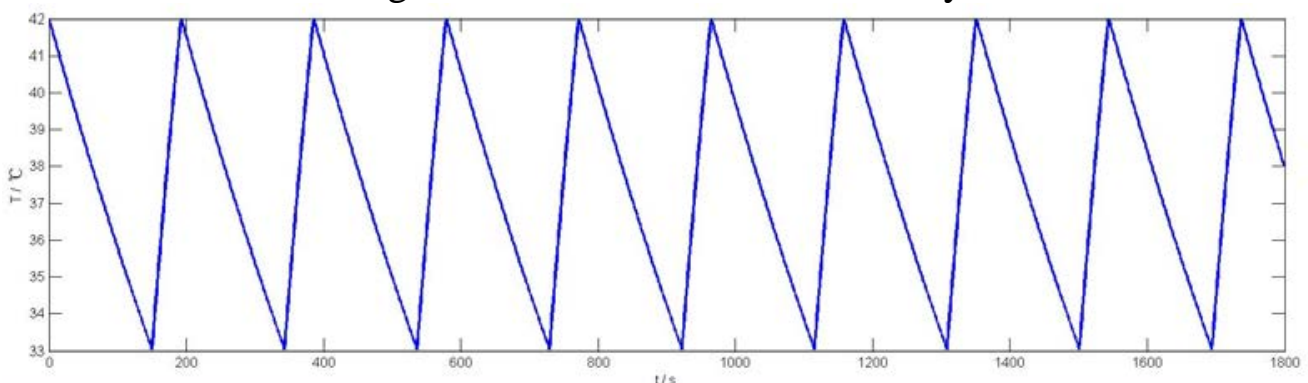

Fig. 1 The water cycle period of the first case

Next, we add hot water when the water temperature comes down to Tc ( $T_{c}>T_{c \min }$ ), which is not the minimum temperature. It's natural that the association between water temperature of bathtub and time is the same with Eq.. By the equation, we obtain that,

$$
t=-\frac{m_{0}}{q} \ln \left(\frac{T_{s}-T_{h}}{T_{c}-T_{h}}\right)=\frac{m_{0}}{q} \ln \left(\frac{42 e^{-0.0016 t}-T_{h}}{T_{s}-T_{h}}\right)+t_{c}
$$

So the expression of the total quality of hot water is

$$
m_{1}=N q\left(t-t_{c}\right)=\left(\frac{t_{t}-t_{c}}{t}\right) q\left(t-t_{c}\right)
$$

where we let tt represent the total time of the hot bath. In the last, we calculate that there exists a minimum value when $t_{c}=116 \mathrm{~s}, m_{1 \min }=249 \mathrm{Kg}$.

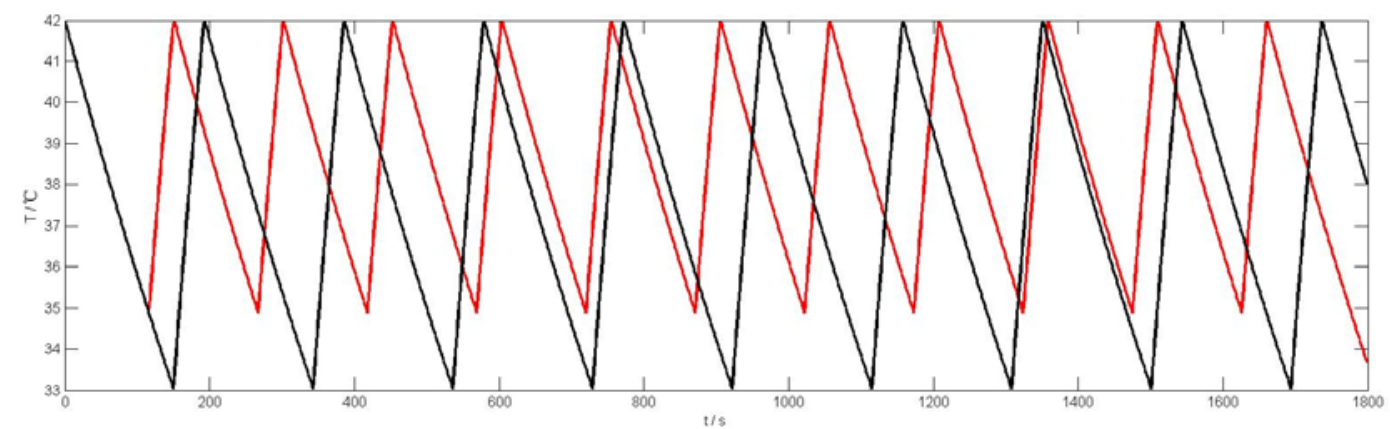

Fig. 2 The contrast of two cases(Red line is shown the second case) 


\section{Conclusion}

Combining these two results we see that, for the first case shown in Fig. 1 is wasting more hot water to maintain the initial temperature than the second case which is shown in Fig. 2. The result is encouraging- it means that under the demand of balancing the temperature with little hot water as much as possible, we can always avoid adding hot water at the moment of the minimum temperature.

\section{References}

[1] Zhang HX. 3D Heating Model of Water Temperature and Researching Control Algorithm in Aquaculture [D]:Northeastern University, 2011.

[2] Lou, HY, Liu, SX. Optimal selection of the body volume calculative model in male college students[J]. Chinese Journal of Clinical Rehabilitation, 2005, 9(40): 134-135. 